\title{
An introductory approach to the concept of spatial coherence
}

H. Rabal, N. Cap, E. Grumel, M. Trivi

H. J. Rabal, N. L. Cap, E. Grumel, M. Trivi, "An introductory approach to the concept of spatial coherence," Proc. SPIE 9289, 12th Education and Training in Optics and Photonics Conference, 92890J (17 July 2014); doi:

$10.1117 / 12.2070781$

SPIE Event: 12th Education and Training in Optics and Photonics Conference, 2013, Porto, Portugal 


\title{
An introductory approach to the concept of spatial coherence
}

\author{
H. J. Rabal, N. L. Cap, E. Grumel, M. Trivi
}

Centro de Investigaciones Ópticas (CONICET La Plata - CIC) and UID Optimo, Departamento Ciencias Básicas, Facultad de Ingeniería, Universidad Nacional de la Plata, P.O. Box 3, Gonnet, La Plata 1897, Argentina

\begin{abstract}
The concept of spatial coherence is usually hard to be understood the first time that it is studied. We propose here a geometric description that does not contain mathematical difficulties and permits to understand how a Young's Fringes system is obtained with a source not spatially coherent. It is based in a very simple experiment that permits the detection of spatial coherence in a scene. Experimental results are shown
\end{abstract}

Keywords: optical coherence, spatial coherence, Van Cittert - Zernike Theorem.

Address all correspondence to: H. J. Rabal, Departamento Ciencias Básicas, Facultad de Ingeniería, Universidad Nacional de la Plata, P.O. Box 3, Gonnet, La Plata 1897, Argentina. Tel: +54 221-484-2957; Fax: +54 221-4712771; E-mail: h_rabal_geb@yahoo.com

\section{INTRODUCTION}

The concept of spatial coherence is usually difficult to understand. The rigorous study of the spatial coherence has been developed by Van Cittert and Zernike [1]. It states that the normalized degree of coherence is the Fourier transform of the intensity distribution for uncorrelated emitters.

When the interference phenomenon is studied, it usually starts with the calculation of the irradiance in an observation plane due to the superposition of two waves.

In this calculation, the irradiance is found to be [2]:

$$
I=2 I_{0}\left(1+\cos \delta_{12}\right)=4 I_{0} \cos ^{2}\left(\frac{\delta_{12}}{2}\right)
$$

Where $I_{0}$ are the irradiance of the two waves and $\delta_{12}$ is the phase difference between them. We assumed that the electrical fields of both waves are parallel. 
The phase difference $\delta_{12}$ in the case of incoherent sources is not constant but changes so fast and ordinary detectors cannot detected the interference phenomenon. The irradiance due to two such sources is the sum of the irradiances of each of them.

Nevertheless, when we observe a light source through a pupil composed by two thin slits, we find a fringe system. Why does it happen? How can we justify it?

We suggest here a description using elementary trigonometric identities to explain how a Young Fringes system can be obtained from a source constituted by incoherent point sources. The visibility in the fringes with a compound source is found as the coincidence of several shifted fringes systems coming each from every single point. These are added on an intensity basis.

This approach leads in a natural way to the same result for the visibility as the Van CittertZernike Theorem for any arbitrary source distribution.

We use a simple experiment to illustrate this proposal. It is consisting in the observation of a scene through a card with two very small and very close slits.

\section{SIMPLE EXPERIMENT: ONLY ONE POINT LIKE LIGHT SOURCE}

If we observe a point like light source with an optical system limited by two parallel slit apertures, it can be observed that in the image there is Young's Fringes fringe pattern.

If the source is composed by several incoherent emitters, the observable irradiance are too fast to be detected in the optical range, their average is zero and no fringes are observed.

Nevertheless, if this optical system is pointed to any natural scene, it can be that it appears covered with fringes. As an example, in Figure 1a) we show a border of a piece of glass. In Figures 1b) and Figure 1c) two examples of the corresponding Young Fringes that can be observed.

How we can solve this contradiction? Why we find fringes? 
To look for the reason we are going to consider very simple sources and to ask what happens with the fringes that they give rise.

\section{TWO POINT SOURCES MUTUALLY NON COHERENT}

In Figure 2, A and B in plane $\pi$ represent two quasi monochromatic point sources separated by a distance $X_{0}$. They have the same wavelength $\lambda$ and the same irradiance $I_{0}$. Narrow slits $P_{1}$ and $P_{2}$ are separated a distance $d$. The lens L, with focal distance $f_{0}$ conjugates the planes $\pi$ and $\pi^{\prime}$. The distance $z$ is much bigger than $d$ distance and the distance $z^{\prime} \sim f_{0}$.

For each quasi monochromatic source corresponds in plane $\pi$ to the light distribution found in a Young's Fringes experiment.

The irradiance distributions $I_{A}$ and $I_{B}$ in plane due to the sources A and B respectively can be described as:

$$
\begin{aligned}
& I_{A}(x)=4 I_{0} \cos ^{2}\left[\frac{\pi}{\lambda} \frac{d}{f_{0}} x\right] \\
& I_{B}(x)=4 I_{0} \cos ^{2}\left[\frac{\pi}{\lambda} \frac{d}{f_{0}}\left(x-X_{0}^{\prime}\right)\right]
\end{aligned}
$$

Following the approach of Rabal [3], the visibility of the fringes system produced by $I_{A}$ and $I_{B}$ is:

$$
V=\frac{I_{M A X}-I_{M I N}}{I_{M A X}+I_{M I N}}=\left|\cos \left(\frac{\pi X^{\prime}{ }_{0} d}{\lambda z^{\prime}}\right)\right|
$$

The visibility depends on the separation $d$ between $\mathrm{P}_{1}$ and $\mathrm{P}_{2}$ (points that are used in the correlation in the Van Cittert Zernike's Theorem) as well as the relation between $\frac{X_{0}^{\prime}}{z^{\prime}}$.

Imposing a $2 \pi$ (or integer multiples) shift between both fringes systems, they will be in consonance and the visibility of the composed system will be a maximum. It is due to 
coincidence of the fringes and not to interference between light coming from the different sources.

Extending the Rabal approach [3] for the case of a continuous quasi monochromatic incoherent intensity distribution source, the visibility of the Young fringes systems becomes:

$$
V=\frac{\left\{\left|\int I\left(x^{\prime}\right) \cos \left[2 w_{x} x^{\prime}\right] d x^{\prime}\right|^{2}+\left|\int I\left(x^{\prime}\right) \sin \left[2 w_{x} x^{\prime}\right] d x^{\prime}\right|^{2}\right\}^{1 / 2}}{\int I\left(x^{\prime}\right) d x^{\prime}}
$$

Where

$$
w_{x}=\frac{\pi}{\lambda} \frac{d}{f_{0}}
$$

The visibility is given by the modulus of the normalized Fourier Transform of the intensity distribution of the source. This is the result of the Van Cittert Zernike's Theorem.

\section{FIGURES}

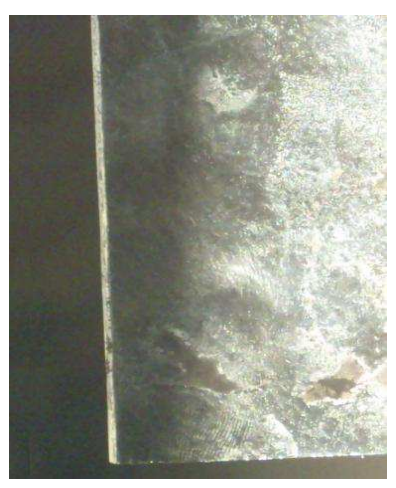

Figure 1a)

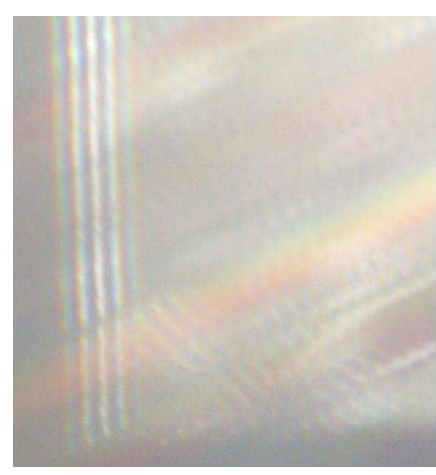

Figure 1b)

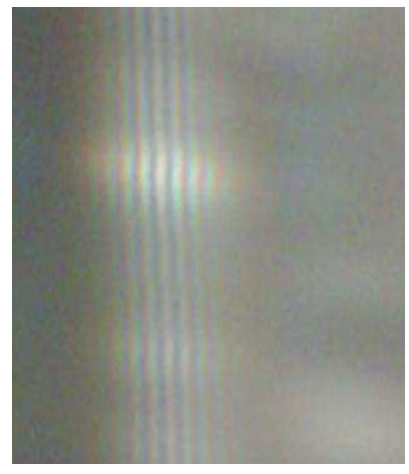

Figure 1c)

Figure 1: a) a border of a piece of glass, b) and c) examples of the corresponding Young fringes. 


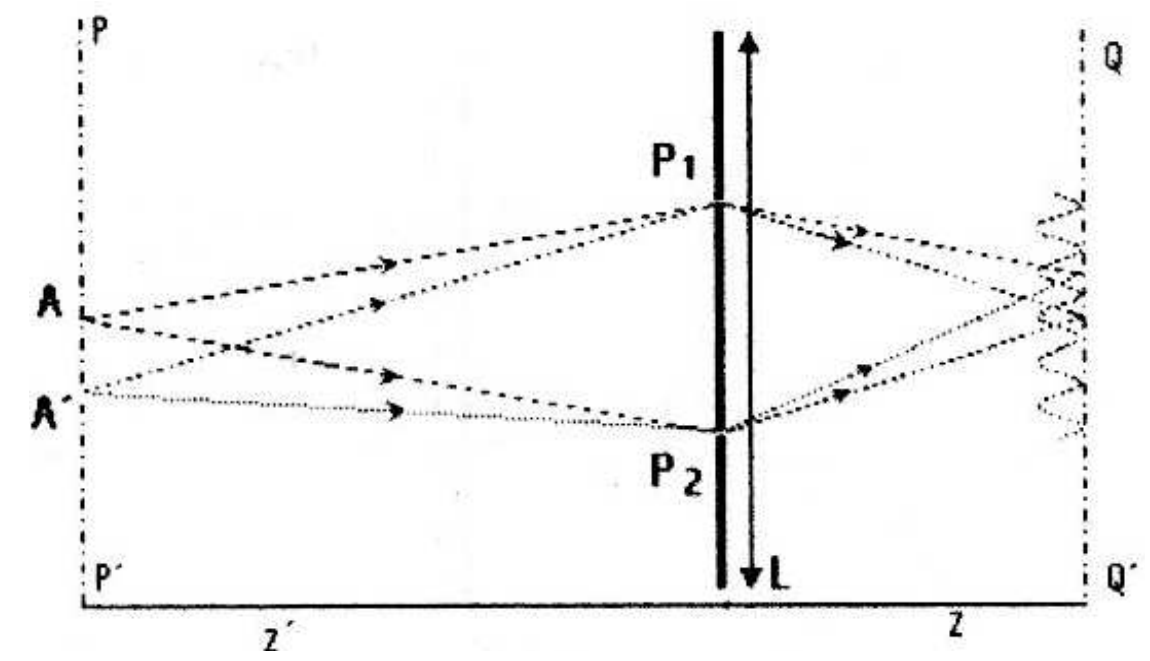

Figure 2. Young fringes pattern obtained from two point sources mutually non coherent.

\section{CONCLUSIONS}

When a single point-like source is observed through a two narrow slits, we obtain a Young fringes pattern, where the contributions of each aperture are added on a field basis.

For the spatial extended light sources, high visibility Young fringes can still be observed if every point of it gives rise to a fringes system that coincides with the origined by the others. In this case the addition of these elementary contributions is in intensity.

For the systems to coincide and obtaining a good visibility result there should not exist source points very near that spoil the others visibility. This only happens when the source exhibits spatial discontinuities (i.e. when the Fourier Transform is not a Dirac's Delta distribution). It is the presence of source discontinuities that gives rise to the visibility predicted by the Van CittertZernike Theorem.

This is also true when the source is a uniform field and there are isolated discontinuities (dilute dark object on bright uniform field). So that visibility behaves as fulfilling a Babinet like complementarity property. 
6. ACKNOWLEDGEMENTS

This research was supported by grants of University of La Plata, ANPCyT, CONICET and CICPBA (Argentina)

\section{REFERENCES}

[1] Born M. and Wolf E. Principles of Optics, (4th.ed.) Pergamon Press, Oxford UK pag. 508. (1970).

[2] Hecht E. and Zajac, A. Optics, Addison Wesley Publishing, Reading MA, (1974)

[3] Rabal, H. "The holodiagram in a geometrical approach to the calculation of fringe visibility". Optik, 113, 260-266, (2002). 